Article

\title{
E-Bayesian Estimation Based on Burr-X Generalized Type-II Hybrid Censored Data
}

\author{
Abdalla Rabie ${ }^{1,2, *(\mathbb{D})}$ and Junping $\mathrm{Li}^{1}$ \\ 1 Central South University, School of Mathematics and Statistics, Changsha 410083, Hunan, China; \\ jpli@csu.edu.cn \\ 2 Department of Mathematics, Faculty of Science, Al-Azhar University, Assiut 71524, Egypt \\ * Correspondence: abuda_math@csu.edu.com; Tel.: +86-155-7488-3297
}

Received: 22 February 2019; Accepted: 22 April 2019; Published: 3 May 2019

\begin{abstract}
In this article, we are concerned with the E-Bayesian (the expectation of Bayesian estimate) method, the maximum likelihood and the Bayesian estimation methods of the shape parameter, and the reliability function of one-parameter Burr-X distribution. A hybrid generalized Type-II censored sample from one-parameter Burr-X distribution is considered. The Bayesian and E-Bayesian approaches are studied under squared error and LINEX loss functions by using the Markov chain Monte Carlo method. Confidence intervals for maximum likelihood estimates, as well as credible intervals for the E-Bayesian and Bayesian estimates, are constructed. Furthermore, an example of real-life data is presented for the sake of the illustration. Finally, the performance of the E-Bayesian estimation method is studied then compared with the performance of the Bayesian and maximum likelihood methods.
\end{abstract}

Keywords: maximum likelihood estimation; Bayesian and E-Bayesian approaches; generalized hybrid censoring scheme; MCMC method; confidence and credible intervals

\section{Introduction}

Hybrid censoring scheme (HCS) is introduced by [1] as a mixture of Type-I and Type-II censoring schemes. In such schemes, experiments are stopped as soon as a pre-specified number $r$ of items from $n$ items fails or when a pre-fixed time $T$ runs out. Thus, there have been two types of censored schemes, namely:

Type-I HCS: in which we terminate the test at $T_{*}=\min \left\{Y_{k: n}, T\right\}$, where $Y_{k: n}$, is the time of $k$-th item failure, $T$ is the maximum time point for the test and $k \in\{1,2, \cdots, n\}$ and $T \in(0, \infty)$ are determined in advance, i.e., the experiment is terminated when a number $k$ failures out of $n$ items or a pre-fixed time $T$ is reached. Type-II HCS: in this type, the experiment is terminated at $T^{*}=\max \left\{Y_{k: n}, T\right\}$, i.e., we end the experiment when the latter of the stopping rules is reached. Hence, we guarantee that at least $k$ failures are obtained.

There are advantages and disadvantages in these schemes. HCS Type-I possesses the advantage of having a fixed time. However, there are a small number of failures obtained before the stopping time of the life-testing. These disadvantages may impact the accuracy of estimators. However, a drawback of Type-I HCS is overcome by Type-II HCS, and we guarantee The obtaining of a specified number of failures. It also has a disadvantage in that the experimenter cannot know when the required specified failures are observed. Ref. [2] proposed two generalized HCSs to overcome these drawbacks, described as follows. Generalized Type-I HCS: Let $r, k \in\{1,2, \cdots, n\}, k<r<n$, and time $T \in(0, \infty)$. When $k$-th failure is obtained before time $T$, the experiment is terminated at $\min \left\{Y_{r: n}, T\right\}$. If the $k$-th failure is obtained after time $T$, the experiment is ended at $Y_{k: n}$. 
Generalized Type-II HCS: Fix $r \in\{1,2, \cdots, n\}$ and $T_{1}, T_{2} \in(0, \infty)$ where $T_{1}<T_{2}$. The experiment is terminated at $T_{1}$, if $r$-th failure occurs before $T_{1}$. When the $r$-th failure occurs between $T_{1}$ and $T_{2}$, we terminate at $Y_{r: n}$. In the third case, if $r$-th failure is observed after time $T_{2}$, the experiment is terminated at $T_{2}$. This scheme has been studied by many authors, such as [3], who presented details on censoring scheme developments in addition to generalized and unified HCS. Ref. [4] discussed Bayesian analysis and prediction based on generalized Type-II HCS for exponential and Pareto models. Ref. [5] studied maximum likelihood, Bayes and percentile bootstrap methods for unknown parameters, failure rate function, the survival function and the coefficient of variation of the exponential Rayleigh distribution with generalized Type-II HCS.

Here, generalized Type-II HCS is considered. We observe one of these types of the censored data.

Case 1: $\left\{Y_{1: n}<Y_{2: n}<\cdots<Y_{r: n}<\cdots<Y_{d 1: n}<T_{1}\right\}$ if $Y_{r: n}<\cdots<Y_{d 1: n}<T_{1}$. In this case, the $r$-th failure is obtained before $T_{1}$, so the experiment is stopped at $T_{1}$ and $d_{1}$ number of failures is obtained at time $T_{1}$.

Case 2: $\left\{Y_{1: n}<Y_{2: n}<\cdots<Y_{d 1: n}<\cdots<Y_{r: n}\right\}$ if $T_{1}<Y_{r: n}<T_{2}$. In this case, the $r$-th failure occurs after $T_{1}$, so the experiment is terminated at $Y_{r: n}$, and $r$ number of failures is obtained.

Case 3: $\left\{Y_{1: n}<Y_{2: n}<\cdots<Y_{d 2: n}<T_{2}\right\}$ if $Y_{r: n}>T_{2}$. In this case, the $r$-th failure occurs after $T_{2}$, so the experiment is ended at $T_{2}$ and $d_{2}$ number of failures is obtained at time $T_{2}$. where $T_{1}$ and $T_{2}$ are time points determined by the experimenter according to how the experiment should continue based on the information about the product.

\section{Burr-X as a Lifetime Model}

Burr-X model is part of Burr distribution family suggested by [6]. This distribution is important in many fields such as operations research and statistics. It is widely used in health, agriculture, and biology. For more details on the applications of this model, one can refer to [7], as they discussed the cumulative distribution function (CDF) of Burr-X distribution with the cumulative damage process and the shock model. Also, they assumed a mathematical model for the expected lifetime of AIDS patients, then fitted the observed data of infected persons for Burr-X distribution. The probability density function (PDF) of one-parameter Burr- $\mathrm{X}$ distribution is given by

$$
f(y ; \theta)=2 \theta y e^{-y^{2}}\left(1-e^{-y^{2}}\right)^{\theta}, y>0, \theta>0,
$$

and the CDF takes the form

$$
F(y ; \theta)=\left(1-e^{-y^{2}}\right)^{\theta}, y>0, \theta>0,
$$

where $\theta$ is the shape parameter. The reliability function $R(t)$ and the hazard rate function $h(t)$ for one-parameter Burr- $X$ distribution are, respectively, expressed as follows:

$$
\begin{gathered}
R(t)=1-\left(1-e^{-t^{2}}\right)^{\theta}, t>0, \\
h(t)=\frac{2 \theta t e^{-t^{2}}\left(1-e^{-t^{2}}\right)^{\theta-1}}{1-\left(1-e^{-t^{2}}\right)^{\theta}}, t>0 .
\end{gathered}
$$

Many publications discuss Burr-X distribution, such as [8], who discussed maximum likelihood estimate (MLE) and Bayesian estimates for parameters of Burr-X model under double Type-II censored sample of dual generalized order statistics. Ref. [9] considered the Bayesian and non-Bayesian estimators of the parameter of the Burr-X model based on grouped data. Ref. [10] studied inferences and predictions for the Burr Type-X model based on records. Ref. [11] considered the statistical analysis of the parameter through different priors and loss functions. Ref. [12] discussed E-Bayesian method and ML and Bayesian methods for the parameter and the reliability function of the Burr-X model under Type-I HCS. 
We assume $Y_{1: n}, Y_{2: n}, \cdots, Y_{n: n}$ are $n$ lifetimes of failure observations under a generalized Type-II hybrid censored sample following one-parameter Burr- $X$ distribution. Let $D_{1}$ and $D_{2}$ denote several items that fail before $T_{1}$ and $T_{2}$, respectively; the likelihood function for three cases is given by

$$
L(\theta)= \begin{cases}\frac{(2 \theta)^{D_{1}} n !}{\left(n-D_{1}\right) !}\left\{1-W_{T_{1}}^{\theta}\right\}^{n-D_{1}} \prod_{i=1}^{D_{1}} y_{i: n} e^{-y_{i: n}^{2}} W_{y_{i: n}}^{\theta-1}, & D_{1}=r, r+1, \cdots, n, \\ \frac{(2 \theta)^{r} n !}{(n-r) !}\left\{1-W_{y_{r: n}}^{\theta}\right\}^{n-r} \prod_{i=1}^{r} y_{i: n} e^{-y_{i: n}^{2}} W_{y_{i: n}}^{\theta-1}, & D_{1}=0,1, \cdots, r-1, D_{2}=r \\ \frac{(2 \theta)^{D_{2} n !}}{\left(n-D_{2}\right) !}\left\{1-W_{T_{2}}^{\theta}\right\}^{n-D_{2}} \prod_{i=1}^{D_{2}} y_{i: n} e^{-y_{i: n}^{2}} W_{y_{i: n}}^{\theta-1}, & D_{2}=0,1, \cdots, r-1 .\end{cases}
$$

where $W_{T_{1}}=1-e^{-T_{1}^{2}}, W_{y_{i: n}}=1-e^{-y_{i: n}^{2}}$ and $W_{T_{2}}=1-e^{-T_{2}^{2}}$.

By taking the natural logarithm of Equation (5), ignoring the constant term, as it does not depend on $\theta$, we get the log-likelihood function as follows:

$$
\ell=\ln L(\theta)=\left\{\begin{array}{l}
D_{1} \ln (2 \theta)+\left(n-D_{1}\right) \ln \left(1-W_{T_{1}}^{\theta}\right)+\sum_{i=1}^{D_{1}} \ln y_{i: n}-\sum_{i=1}^{D_{1}} y_{i: n}^{2}+(\theta-1) \ln W_{y_{i: n},} \\
D_{1}=r, r+1, \cdots, n, \\
r \ln (2 \theta)+(n-r) \ln \left(1-W_{y_{r: n}}^{\theta}\right)+\sum_{i=1}^{r} \ln y_{i: n}-\sum_{i=1}^{r} y_{i: n}^{2}+(\theta-1) \ln W_{y_{i: n},} \\
D_{1}=0,1, \cdots, r-1, D_{2}=r, \\
D_{2} \ln (2 \theta)+\left(n-D_{2}\right) \ln \left(1-W_{T_{2}}^{\theta}\right)+\sum_{i=1}^{D_{2}} \ln y_{i: n}-\sum_{i=1}^{D_{2}} y_{i: n}^{2}+(\theta-1) \ln W_{y_{i: n},} \\
D_{2}=0,1, \cdots, r-1 .
\end{array}\right.
$$

The MLE of the unknown parameter $\theta$ is obtained by differentiating Equation (6) regarding $\theta$ then equating the derivative to zero (see Appendix A) then solving numerically the following equation:

$$
\frac{\partial \ell}{\partial \theta}=0
$$

we get $\hat{\theta}$, the MLE of the parameter $\theta$. Also, we can obtain $R \hat{(t)}$ the MLE of $R(t)$ by replacing $\theta$ with $\hat{\theta}$ in Equation (3), as follows:

$$
R \hat{(t)}=1-\left(1-e^{-t^{2}}\right)^{\hat{\theta}}
$$

The rest of the article is presented as follows. The Bayesian estimation under SEL and LINEX functions is studied in Section 2. Section 3 discusses the E-Bayesian method under SEL and LINEX loss functions. The Markov chain Monte Carlo (MCMC) algorithm is presented in Section 4. Section 5 provides an example of real-life data sets. The results are compared and concluded in Section 6.

\section{Bayesian Estimation Method}

Here, the Bayesian estimation of the parameter $\theta$ and the reliability function $R(t)$ of one-parameter Burr- $X$ distribution based on generalized Type-II HCS is considered. The Bayesian approach needs to specify the prior PDF of the parameter $\theta$. The gamma PDFs are widely used in the Bayesian analysis, so we suppose the gamma conjugates prior PDF for $\theta$ (as suggested by $[8,9]$ ) with the PDF of the form.

$$
\pi(\theta)=\frac{b^{a}}{\Gamma(a)} \theta^{a-1} e^{-b \theta}, a, b>0,
$$


the posterior PDF of $\theta$ can be given from (5) and (9), as follows:

$$
g(\theta \mid y)= \begin{cases}K^{-1} \theta^{D_{1}+a-1} e^{-b \theta}\left\{1-W_{T_{1}}^{\theta}\right\}^{n-D_{1}} \prod_{i=1}^{D_{1}} y_{i: n} e^{-y_{i: n}^{2}} W_{y_{i: n}}^{\theta-1}, & D_{1}=r, r+1, \cdots, n, \\ K^{-1} \theta^{r+a-1} e^{-b \theta}\left\{1-W_{y_{r: n}}^{\theta}\right\}^{n-r} \prod_{i=1}^{r} y_{i: n} e^{-y_{i: n}^{2}} W_{y_{i: n}}^{\theta-1}, & D_{1}=0,1, \cdots, r-1, D_{2}=r \\ K^{-1} \theta^{D_{2}+a-1} e^{-b \theta}\left\{1-W_{T_{2}}^{\theta}\right\}^{n-D_{2}} \prod_{i=1}^{D_{2}} y_{i: n} e^{-y_{i: n}^{2}} W_{y_{i: n}}^{\theta-1}, & D_{2}=0,1, \cdots, r-1 .\end{cases}
$$

where $K$ is the normalizing constant given as

$$
K=\int_{0}^{\infty} g(\theta \mid y) d \theta .
$$

\subsection{Estimates under Squared Error Loss Function}

The Bayesian estimate of $\theta$ under SEL function is given by

$$
\begin{aligned}
\hat{\theta}_{B S} & =E[\theta \mid y] \\
& =\int_{0}^{\infty} \theta g(\theta \mid y) d \theta \\
& = \begin{cases}K^{-1} \int_{0}^{\infty} \theta^{D_{1}+a} e^{-b \theta}\left\{1-W_{T_{1}}^{\theta}\right\}^{n-D_{1}} \prod_{i=1}^{D_{1}} y_{i: n} e^{-y_{i: n}^{2}} W_{y_{i: n}}^{\theta-1} d \theta, & D_{1}=r, r+1, \cdots, n, \\
K^{-1} \int_{0}^{\infty} \theta^{r+a} e^{-b \theta}\left\{1-W_{y_{r: n}}^{\theta}\right\}^{n-r} \prod_{i=1}^{r} y_{i: n} e^{-y_{i: n}^{2}} W_{y_{i: n}}^{\theta-1} d \theta, & D_{1}=0,1, \cdots, r-1, D_{2}=r, \\
K^{-1} \int_{0}^{\infty} \theta^{D_{2}+a} e^{-b \theta}\left\{1-W_{T_{2}}^{\theta}\right\}^{n-D_{2}} \prod_{i=1}^{D_{2}} y_{i: n} e^{-y_{i: n}^{2}} W_{y_{i: n}}^{\theta-1} d \theta, & D_{2}=0,1, \cdots, r-1 .\end{cases}
\end{aligned}
$$

The Bayesian estimate for the reliability function $R(t)$ by using the SEL function is given as follows:

$$
\begin{aligned}
\hat{R}(t)_{B S} & =E[R(t) \mid y] \\
& =\int_{0}^{\infty} R(t) g(\theta \mid y) d \theta \\
& =\left\{\begin{array}{l}
K^{-1} \int_{0}^{\infty}\left(1-\left(1-e^{-t^{2}}\right)^{\theta}\right) \theta^{D_{1}+a-1} e^{-b \theta}\left\{1-W_{T_{1}}^{\theta}\right\}^{n-D_{1}} \prod_{i=1}^{D_{1}} y_{i: n} e^{-y_{i: n}^{2}} W_{y_{i: n}}^{\theta-1} d \theta, \\
D_{1}=r, r+1, \cdots, n \\
K^{-1} \int_{0}^{\infty}\left(1-\left(1-e^{-t^{2}}\right)^{\theta}\right) \theta^{r+a-1} e^{-b \theta}\left\{1-W_{y_{r: n}}^{\theta}\right\}^{n-r} \prod_{i=1}^{r} y_{i: n} e^{-y_{i: n}^{2}} W_{y_{i: n}}^{\theta-1} d \theta, \\
D_{1}=0,1, \cdots, r-1, D_{2}=r \\
K^{-1} \int_{0}^{\infty}\left(1-\left(1-e^{-t^{2}}\right)^{\theta}\right) \theta^{D_{2}+a-1} e^{-b \theta}\left\{1-W_{T_{2}}^{\theta}\right\}^{n-D_{2}} \prod_{i=1}^{D_{2}} y_{i: n} e^{-y_{i: n}^{2}} W_{y_{i: n}}^{\theta-1} d \theta,
\end{array}\right.
\end{aligned}
$$

\subsection{Estimates under LINEX Loss Function}

The LINEX loss function with parameters $k, h$ is given by

$$
L(\hat{\theta}, \theta)=k\left\{e^{h(\hat{\theta}-\theta)}-h(\hat{\theta}-\theta)-1\right\},
$$


where $\hat{\theta}$ is the estimate of $\theta$ and $h$ is the shape parameter of loss function. For more details, see [13]. The Bayesian estimate $\hat{\theta}_{B L}$ of the parameter $\theta$ using the LINEX loss function is $\hat{\theta}$, which minimizes Equation (13) given by

$$
\begin{aligned}
\hat{\theta}_{B L} & =\frac{-1}{h} \ln E[\exp (-h \theta) \mid y] \\
& =\frac{-1}{h} \ln \int_{0}^{\infty} \exp (-h \theta) g(\theta \mid y) d \theta \\
& =\left\{\begin{array}{l}
\frac{-1}{K h} \ln \int_{0}^{\infty} \theta^{D_{1}+a-1} e^{-(b+h) \theta}\left\{1-W_{T_{1}}^{\theta}\right\}^{n-D_{1}} \prod_{i=1}^{D_{1}} y_{i: n} e^{-y_{i: n}^{2}} W_{y_{i: n}}^{\theta-1} d \theta, \\
D_{1}=r, r+1, \cdots, n, \\
\frac{-1}{K h} \ln \int_{0}^{\infty} \theta^{r+a-1} e^{-(b+h) \theta}\left\{1-W_{y_{r: n}}^{\theta}\right\}^{n-r} \prod_{i=1}^{r} y_{i: n} e^{-y_{i: n}^{2}} W_{y_{i: n}}^{\theta-1} d \theta, \\
D_{1}=0,1, \cdots, r-1, D_{2}=r, \\
\frac{-1}{K h} \ln \int_{0}^{\infty} \theta^{D_{2}+a-1} e^{-(b+h) \theta}\left\{1-W_{T_{2}}^{\theta}\right\}^{n-D_{2}} \prod_{i=1}^{D_{2}} y_{i: n} e^{-y_{i: n}^{2}} W_{y_{i: n}}^{\theta-1} d \theta, \\
D_{2}=0,1, \cdots, r-1, \quad h \neq 0,
\end{array}\right.
\end{aligned}
$$

By using the LINEX loss, the Bayesian estimate of $R(t)$ is given by

$$
\begin{aligned}
R \hat{(t})_{B L}= & \frac{-1}{h} \ln E[\exp (-h R(t)) \mid y] \\
= & \frac{-1}{h} \ln \int_{0}^{\infty} \exp (-h R(t)) g(\theta \mid y) d \theta \\
= & \left\{\begin{array}{l}
=\frac{-1}{K h} \ln \int_{0}^{\infty} \theta^{D_{1}+a-1} e^{h\left[\left(1-\exp \left(-t^{2}\right)\right)^{\theta}-1\right]-b \theta}\left\{1-W_{T_{1}}^{\theta}\right\}^{n-D_{1}} \prod_{i=1}^{D_{1}} y_{i: n} e^{-y_{i: n}^{2}} W_{y_{i: n}}^{\theta-1} d \theta, \\
D_{1}=r, r+1, \cdots, n, \\
=\frac{-1}{K h} \ln \int_{0}^{\infty} \theta^{r+a-1} e^{h\left[\left(1-\exp \left(-t^{2}\right)\right)^{\theta}-1\right]-b \theta}\left\{1-W_{y_{r: n}}^{\theta}\right\}^{n-r} \prod_{i=1}^{r} y_{i: n} e^{-y_{i: n}^{2}} W_{y_{i: n}}^{\theta-1} d \theta, \\
D_{1}=0,1, \cdots, r-1, D_{2}=r, \\
=\frac{-1}{K h} \ln \int_{0}^{\infty} \theta^{D_{2}+a-1} e^{h\left[\left(1-\exp \left(-t^{2}\right)\right)^{\theta}-1\right]-b \theta}\left\{1-W_{T_{2}}^{\theta}\right\}^{n-D_{2}} \prod_{i=1}^{D_{2}} y_{i: n} e^{-y_{i: n}^{2} W_{y_{i: n}}^{\theta-1} d \theta,} \\
D_{2}=0,1, \cdots, r-1, \quad h \neq 0 .
\end{array}\right.
\end{aligned}
$$

\section{E-Bayesian Estimation Method}

The possibility of the rate of failure for high-reliability products is less than the possibility of the failure rate of low-reliability products. For this, according to [14], we select the hyper-parameters $a$ and $b$ to prove that $\pi(\theta)$ is a decreasing function of $\theta$. The first derivative of $\pi(\theta)$ regarding $\theta$ is as follows:

$$
\frac{d \pi(\theta)}{d \theta}=\frac{b^{a}}{\Gamma(a)} \theta^{a-2} \exp (-b \theta)\{(a-1)-b \theta\} .
$$

Thus, for $0<a<1$ and $b>0$, the prior $\operatorname{PDF} \pi(\theta)$ is a decreasing function of $\theta$. The thinnertailed prior PDF often decreases the robustness of the Bayesian estimate; therefore, $b$ should be smaller than an upper bound $c$, where $c>0$ is a constant. Accordingly, hyper-parameters $a$ and $b$ should be selected with the restriction of $0<a<1$ and $0<b<c$. We assume that $a$ and $b$ are independent with bi-variate PDF given by

$$
p(a, b)=p_{1}(a) p_{2}(b)
$$


the E-Bayesian estimates of $\theta$ and $R(t)$ are, respectively, obtained as follows:

$$
\begin{gathered}
\hat{\theta}_{E B}=E\left[\hat{\theta}_{B} \mid y\right]=\iint_{D} \hat{\theta_{B}}(a, b) p(a, b) d a d b, \\
\left.\left.R \hat{(t)})_{E B}=E[R \hat{(t})_{B} \mid y\right]=\iint_{D} R \hat{(t)}\right)_{B} p(a, b) d a d b .
\end{gathered}
$$

where $D$ stands for the set of possible values of $a$ and $b, \hat{\theta}_{B}$ and $\left.R \hat{(t}\right)_{B}$ are the Bayesian estimates of $\theta$ and $R(t)$, respectively, under the SEL and the LINEX loss functions. See, for more details, [15-18].

\subsection{The E-Bayesian Estimate of $\theta$}

To obtain the E-Bayesian estimate of $\theta$, we use three prior PDFs of $a$ and $b$ to study the influence of these prior PDFs on the E-Bayesian estimation. We suggest the following prior PDFs

$$
\begin{array}{ll}
p_{1}(a, b)=\frac{2 a}{c}, & 0<a<1,0<b<c, \\
p_{2}(a, b)=\frac{2 b}{c^{2}}, & 0<a<1,0<b<c, \\
p_{3}(a, b)=\frac{3 b^{2}}{c^{3}}, & 0<a<1,0<b<c,
\end{array}
$$

The E-Bayesian estimate of $\theta$, under the SEL are computed from (11), (16) and (18) as follows:

$$
\hat{\theta}_{E B S_{j}}=\iint_{D} \hat{\theta}_{B S}(a, b) p_{j}(a, b) d a d b, \quad j=1,2,3,
$$

also, under the LINEX loss function, the E-Bayesian estimate of $\theta$, can be derived from (14), (16) and (18) as

$$
\hat{\theta}_{E B L_{j}}=\iint_{D} \hat{\theta}_{B L}(a, b) p_{j}(a, b) d a d b, \quad j=1,2,3 .
$$

\subsection{The E-Bayesian Estimate of $R(t)$}

The E-Bayesian estimate can be computed by adopting (18) based on the SEL and LINEX loss functions as follows.

The E-Bayesian estimate of $R(t)$ under SEL function is written from (12), (17) and (18) as

$$
\left.R \hat{(t)})_{E B S_{j}}=\iint_{D} R \hat{(t}\right)_{B S} p_{j}(a, b) d a d b, \quad j=1,2,3
$$

also, the E-Bayesian estimate of $R(t)$ by using the LINEX loss function can be computed from (15), (17) and (18) as follows:

$$
\left.R \hat{(t})_{E B L_{j}}=\iint_{D} R \hat{(t}\right)_{B L} p_{j}(a, b) d a d b, \quad j=1,2,3 .
$$

It is noted that E-Bayesian and Bayesian estimates cannot be obtained analytically; therefore, we use the MCMC method for deriving the Bayesian and E-Bayesian estimates of $\theta$ and $R(t)$.

\section{The MCMC Algorithm}

In this work, we consider the MCMC method for generating a sample of $\theta$ from the posterior PDF; after that, we derive Bayesian and E-Bayesian estimates of $\theta$ and $R(t)$ under LINEX loss and SEL functions. A Metropolisâ€“ Hastings sampler is used to obtain the posterior sample of the parameter $\theta$ 
from the full conditional posterior PDF. From (10), discarding all terms do not depend on $\theta$; we get the full conditional posterior PDF as follows.

$$
g^{*}(\theta \mid y)= \begin{cases}\theta^{D_{1}+a-1} e^{-b \theta}\left\{-W_{T_{1}}^{\theta}\right\}^{n-D_{1}} \prod_{i=1}^{D_{1}} W_{y_{i: n}}^{\theta-1}, & D_{1}=r, r+1, \cdots, n, \\ \theta^{r+a-1} e^{-b \theta}\left\{-W_{y_{r: n}}^{\theta}\right\}^{n-r} \prod_{i=1}^{r} W_{y_{i: n}}^{\theta-1}, & D_{1}=0,1, \cdots, r-1, D_{2}=r, \\ \theta^{D_{2}+a-1} e^{-b \theta}\left\{-W_{T_{2}}^{\theta}\right\}^{n-D_{2}} \prod_{i=1}^{D_{2}} W_{y_{i: n}}^{\theta-1}, & D_{2}=0,1, \cdots, r-1 .\end{cases}
$$

where $W_{y_{i: n}}=1-e^{-y_{i: n}^{2}}, W_{T_{1}}=1-e^{-T_{1}^{2}}, W_{T_{2}}=1-e^{-T_{2}^{2}}$.

For more details, see, for example, $[19,20]$. The following steps indicate the Metropolisâ€ "Hastings algorithm for simulating the posterior samples, then derive the Bayesian estimates and the credible related intervals (CRIs) as follows.

(1) Set the initial value of $\theta$, say $\theta^{(0)}\left(=\hat{\theta}_{M L E}\right)$, to guarantee a rapid convergence of the Markov chain.

(2) Set $j=1$.

(3) By applying a Metropolisâ€“ Hastings sampler, $\theta^{(j)}$ is simulated from $g^{*}\left(\theta^{(j-1)} \mid y\right)$

- Generate a proposal $\theta^{(*)}$ from a normal distribution as a proposal distribution ignoring negative draws, as they lead to a high rejection rate.

- A sample $\mathrm{u}$ is generated from the Uniform $(0,1)$ distribution.

- Compute the acceptance probability

$$
\psi_{\theta}=\min \left[1, \frac{g^{*}\left(\theta^{(*)} \mid y\right)}{g^{*}\left(\theta^{(j-1)} \mid y\right)}\right] .
$$

- If $u<\psi_{\theta}$ accept $\theta^{(*)}$ as $\theta^{(j)}$, or else, $\theta^{(j)}=\theta^{(j-1)}$

(4) Also, $R(t)$ is computed from Equation (3) as follows.

$$
R^{(j)}(t)=1-\left(1-\exp \left(-t^{2}\right)\right)^{\theta^{(j)}} .
$$

(5) Set $j=j+1$.

(6) Steps (3-6) are repeated $N$ times to get a sequence of the parameter $\theta$ with optional burn-in period.

(7) The Bayesian estimates of $\theta$ and $R(t)$ under SEL function are, respectively, given as

$$
\begin{aligned}
\hat{\theta}_{B S} & =\frac{1}{N-M} \sum_{j=M+1}^{N} \theta^{(j)} . \\
R \hat{(t})_{B S} & =\frac{1}{N-M} \sum_{j=M+1}^{N} R^{(j)}(t),
\end{aligned}
$$

where $M$ represents a burn-in period of Markov chain discarded to get to the rapid convergence and cancel the effect of choosing the starting point of the parameter.

(8) The Bayesian estimates for $\theta$ and $R(t)$ by the LINEX loss function are, respectively, given by,

$$
\begin{gathered}
\hat{\theta}_{B L}=\frac{-1}{h} \ln \left[\frac{1}{N-M} \sum_{j=M+1}^{N} e^{-h \theta^{(j)}}\right], \quad h \neq 0, \\
R \hat{(t)})_{B L}=\frac{-1}{h} \ln \left[\frac{1}{N-M} \sum_{j=M+1}^{N} e^{-h R^{(j)}(t)}\right], \quad h \neq 0 .
\end{gathered}
$$


(9) A 100(1- $\gamma) \%$, confidence intervals (CIs) for MLEs of $\theta$ and $R(t)$ are obtained as follows:

$$
\hat{\theta} \pm z_{\frac{\gamma}{2}}(\sqrt{\operatorname{Var}(\hat{\theta})}) \text { and } R \hat{(t)} \pm z_{\frac{\gamma}{2}}(\sqrt{\operatorname{Var}(R \hat{(t}))})
$$

where $z_{\frac{\gamma}{2}}$ is the $100\left(1-\frac{\gamma}{2}\right) \%$ upper percentile of standard normal variate.

(10) A $100(1-\gamma) \%$, CRIs of E-Bayesian and Bayesian estimates of $\theta$ and $R(t)$ are constructed from the $\left(\frac{\gamma}{2}\right)$ and $\left(1-\frac{\gamma}{2}\right)$ quantiles sample of the empirical posterior PDF of MCMC draws, given by,

$$
\left[\theta_{N\left[\frac{\gamma}{2}\right]}, \theta_{N\left[1-\frac{\gamma}{2}\right]}\right] \text { and }\left[R_{N\left[\frac{\gamma}{2}\right]}, R_{N\left[1-\frac{\gamma}{2}\right]}\right]
$$

where $N$ stands for the number of draws.

\subsection{Simulation Study}

- Choose values of $n, r, T_{1}, T_{2}, h$.

- $\quad$ By specifying the value of $c$, values of $a$ and $b$ are generated from (18).

- For known values of $a$ and $b$, the true value of $\theta$ is generated from gamma $(\mathrm{a}, \mathrm{b})$.

- MLEs of the parameter $\theta$ and the reliability function $R(t)$ are, respectively, obtained from Equations (7) and (8).

- A Metropolisâ€“Hastings sampler is used for generating a Markov chain with 11,000 values of $\theta$, ignoring the first 1000 values as a "burn-in" period of the Markov chain.

- Compute the Bayesian estimates of $\theta$ and $R(t)$ under SEL function from (26) and (27), respectively.

- Also, the Bayesian estimates of the parameter $\theta$ and the reliability function $R(t)$ with the LINEX loss function are, respectively, computed from (28) and (Section 4.1).

- Compute the E-Bayesian estimates of the parameter $\theta$ and the reliability function $R(t)$ under SEL function from (19) and (21), respectively.

- The E-Bayesian estimates of parameter $\theta$ and the reliability function $R(t)$ under LINEX loss function are, respectively, obtained from (20) and (22).

- The $95 \%$ CIs of MLEs of $\theta$ and $R(t)$ are constructed from (30).

- The $95 \%$ CRIs of E-Bayesian and Bayesian estimates of the parameter $\theta$ and the reliability function $R(t)$ are computed from (31).

- The mean squared error (MSE) of $\theta$ and $R(t)$ estimates are, respectively, given by,

$$
\begin{gathered}
\operatorname{MSE}(\hat{\theta})=\frac{1}{1000} \sum_{j=1}^{1000}\left(\hat{\theta}_{j}-\theta\right)^{2} \\
\left.\operatorname{MSE}(R \hat{(t}))=\frac{1}{1000} \sum_{j=1}^{1000}(R \hat{(t})_{j}-R(t)\right)^{2},
\end{gathered}
$$

where $\hat{\theta}$ and $R \hat{(t)}$ denote the estimates of $\theta$ and $R(t)$, respectively. To study the impact of values of $n, r, T_{1}$, and $T_{2}$ on MSEs of estimators, we computed estimates across different values of $n, r, T_{1}$, and $T_{2}$. The numerical results are computed by MATHEMATICA 8 codes, such as (FindRoot, NMaximize, NIntegrate and RandomReal) and listed in Tables 1 and 2, where Table 1, gives estimates, MSE, and 95\% CIs and CRIs for MLE, Bayesian, and E-Bayesian estimates of the parameter $\theta$ with $n=25,45,60,80$; $r=15,30,45,65 ; T_{1}=0.7,1.5$ and $T_{2}=1.5,2$. Table 2 shows average estimates, MSE and 95\% CIs and CRIs of MLE, Bayesian, and E-Bayesian estimates for the reliability function $R(t=1.3)$ with $n=25,45,60,80 ; r=15,30,45,65 ; T_{1}=0.7,1.5$, and $T_{2}=1.5,2$. 
Table 1. Estimates, MSE, and 95\%CIs and CRIs of MLEs, Bayesian, and E-Bayesian methods for $\theta$ under LINEX and SEL functions when $\theta=1.22, a=0.8, b=0.7, h=1.5, c=1$.

\begin{tabular}{|c|c|c|c|c|c|c|c|c|c|c|c|}
\hline \multirow[t]{2}{*}{$(n, r)$} & \multirow[t]{2}{*}{$\left(T_{1}, T_{2}\right)$} & \multirow[t]{2}{*}{ Criteria } & \multirow[t]{2}{*}{$\hat{\theta}_{M L E}$} & \multicolumn{4}{|c|}{ SEL Function } & \multicolumn{4}{|c|}{ LINEX Loss } \\
\hline & & & & $\hat{\boldsymbol{\theta}}_{B S}$ & $\hat{\theta}_{E B S 1}$ & $\hat{\theta}_{E B S 2}$ & $\hat{\theta}_{E B S 3}$ & $\hat{\theta}_{B L}$ & $\hat{\theta}_{E B L 1}$ & $\hat{\theta}_{E B L 2}$ & $\hat{\theta}_{E B L 3}$ \\
\hline \multirow[t]{10}{*}{$(25,15)$} & $(0.7,1.5)$ & Mean & 1.82415 & 1.81515 & 1.62638 & 1.42308 & 1.49423 & 1.72759 & 1.55551 & 1.36835 & 1.43408 \\
\hline & & MSE & 0.56645 & 0.39228 & 0.19571 & 0.06464 & 0.10101 & 0.28806 & 0.13755 & 0.04162 & 0.06726 \\
\hline & & Lower & 0.89686 & 1.1236 & 1.0148 & 0.8420 & 0.3892 & 0.5593 & 0.6968 & 0.2524 & 0.4161 \\
\hline & & Upper & 2.75144 & 2.5067 & 2.4404 & 2.4107 & 2.457 & 2.4291 & 2.4142 & 2.4843 & 2.452 \\
\hline & & Length & 1.85458 & 1.3831 & 1.4256 & 1.5687 & 2.0678 & 1.8698 & 1.7174 & 2.2318 & 2.0359 \\
\hline & $(1.5,2)$ & Mean & 1.88381 & 1.72198 & 1.54289 & 1.35003 & 1.41753 & 1.64524 & 1.48081 & 1.30211 & 1.36485 \\
\hline & & MSE & 0.49302 & 0.2907 & 0.13534 & 0.04071 & 0.06526 & 0.21197 & 0.09359 & 0.0268 & 0.04291 \\
\hline & & Lower & 1.41099 & 1.0782 & 0.9838 & 0.8095 & 0.3772 & 0.5395 & 0.682 & 0.2571 & 0.4137 \\
\hline & & Upper & 2.35663 & 2.3658 & 2.3067 & 2.2763 & 2.3229 & 2.2956 & 2.2796 & 2.3471 & 2.316 \\
\hline & & Length & 0.945648 & 1.2876 & 1.3229 & 1.4668 & 1.9457 & 1.7561 & 1.5975 & 2.09 & 1.9022 \\
\hline \multirow[t]{10}{*}{$(45,30)$} & $(0.7,1.5)$ & Mean & 1.81167 & 1.77186 & 1.58758 & 1.38913 & 1.45859 & 1.72116 & 1.5467 & 1.35768 & 1.42397 \\
\hline & & MSE & 0.56434 & 0.32622 & 0.15252 & 0.04193 & 0.07161 & 0.27055 & 0.12248 & 0.03116 & 0.0550 \\
\hline & & Lower & 0.855328 & 1.2527 & 1.1926 & 0.9552 & 0.4769 & 0.6545 & 0.8653 & 0.3941 & 0.5670 \\
\hline & & Upper & 2.76801 & 2.291 & 2.2498 & 2.220 & 2.3014 & 2.2627 & 2.2281 & 2.3213 & 2.2810 \\
\hline & & Length & 1.91268 & 1.0382 & 1.0572 & 1.2648 & 1.8245 & 1.6081 & 1.3628 & 1.9272 & 1.714 \\
\hline & $(1.5,2)$ & & 1.76545 & 1.68398 & 1.50884 & 1.32024 & 1.38625 & 1.63871 & 1.47236 & 1.29218 & 1.35537 \\
\hline & & MSE & 0.39961 & 0.2318 & 0.0967 & 0.02021 & 0.03884 & 0.18977 & 0.07545 & 0.01435 & 0.02835 \\
\hline & & Lower & 1.10533 & 1.1955 & 1.1421 & 0.9117 & 0.4559 & 0.6251 & 0.8315 & 0.3820 & 0.5469 \\
\hline & & Upper & 2.42557 & 2.1725 & 2.1353 & 2.1060 & 2.1846 & 2.1474 & 2.1132 & 2.2024 & 2.1638 \\
\hline & & Length & 1.32023 & 0.9770 & 0.9931 & 1.1942 & 1.7286 & 1.5222 & 1.2817 & 1.8204 & 1.6168 \\
\hline \multirow[t]{10}{*}{$(60,45)$} & $(0.7,1.5)$ & Mean & 1.83636 & 1.65933 & 1.48676 & 1.30091 & 1.36596 & 1.62674 & 1.46051 & 1.28075 & 1.34376 \\
\hline & & MSE & 0.41976 & 0.2004 & 0.07709 & 0.01109 & 0.02631 & 0.17223 & 0.06335 & 0.00794 & 0.01999 \\
\hline & & Lower & 1.4239 & 1.2453 & 1.2077 & 0.952 & 0.4854 & 0.6573 & 0.8919 & 0.4310 & 0.5994 \\
\hline & & Upper & 2.24882 & 2.0734 & 2.0457 & 2.0215 & 2.1164 & 2.0746 & 2.0291 & 2.1305 & 2.0881 \\
\hline & & Length & 0.824922 & 0.8281 & 0.8380 & 1.0694 & 1.6310 & 1.4173 & 1.1373 & 1.6996 & 1.4888 \\
\hline & $(1.5,2)$ & Mean & 1.62609 & 1.59426 & 1.42846 & 1.2499 & 1.3124 & 1.56302 & 1.40329 & 1.23055 & 1.29109 \\
\hline & & MSE & 0.18972 & 0.15881 & 0.0585 & 0.01241 & 0.02124 & 0.13462 & 0.04734 & 0.01076 & 0.01674 \\
\hline & & Lower & 1.28096 & 1.190 & 1.1541 & 0.9097 & 0.4630 & 0.6277 & 0.8523 & 0.4109 & 0.5723 \\
\hline & & Upper & 1.97122 & 1.9985 & 1.9719 & 1.9473 & 2.0368 & 1.9971 & 1.9543 & 2.0502 & 2.0099 \\
\hline & & Length & 0.690261 & 0.8085 & 0.8178 & 1.0376 & 1.5738 & 1.3694 & 1.1019 & 1.6392 & 1.4375 \\
\hline \multirow[t]{10}{*}{$(80,65)$} & $(0.7,1.5)$ & Mean & 1.62173 & 1.64368 & 1.47274 & 1.28865 & 1.35308 & 1.61869 & 1.45263 & 1.27321 & 1.33607 \\
\hline & & MSE & 0.2717 & 0.18689 & 0.06981 & 0.00925 & 0.02272 & 0.16581 & 0.05966 & 0.00711 & 0.01818 \\
\hline & & Lower & 0.893912 & 1.2825 & 1.2542 & 0.9801 & 0.5046 & 0.6786 & 0.9324 & 0.4622 & 0.6332 \\
\hline & & Upper & 2.34955 & 2.0048 & 1.9832 & 1.9654 & 2.0727 & 2.0275 & 1.9729 & 2.0842 & 2.0389 \\
\hline & & Length & 1.45564 & 0.7223 & 0.7290 & 0.9853 & 1.5681 & 1.3489 & 1.0405 & 1.6220 & 1.4057 \\
\hline & $(1.5,2)$ & Mean & 1.53225 & 1.63107 & 1.46144 & 1.27876 & 1.34269 & 1.60709 & 1.44215 & 1.26396 & 1.3264 \\
\hline & & MSE & 0.1890 & 0.17092 & 0.05986 & 0.00465 & 0.01637 & 0.15162 & 0.05079 & 0.00305 & 0.01255 \\
\hline & & Lower & 0.869392 & 1.2779 & 1.2508 & 0.9763 & 0.5031 & 0.6762 & 0.9304 & 0.4624 & 0.6326 \\
\hline & & Upper & 2.19511 & 1.9843 & 1.9634 & 1.9465 & 2.0544 & 2.0092 & 1.9539 & 2.0655 & 2.0202 \\
\hline & & Length & 1.32572 & 0.7064 & 0.7126 & 0.9702 & 1.5513 & 1.3331 & 1.0235 & 1.6031 & 1.3876 \\
\hline
\end{tabular}

Where $\hat{\theta}_{E B S 1}, \hat{\theta}_{E B S 2}$ and $\hat{\theta}_{E B S 3}$ are the E-Bayesian estimates of $\theta$ relative to SEL based on $p_{1}(a, b), p_{2}(a, b)$ and $p_{3}(a, b)$, respectively, $\hat{\theta}_{E B L 1}, \hat{\theta}_{E B L 2}$ and $\hat{\theta}_{E B L 3}$ are the E-Bayesian estimates of $\theta$ relative to LINEX based on $p_{1}(a, b), p_{2}(a, b)$ and $p_{3}(a, b)$, respectively. In addition, $\hat{\theta}_{B S}$ and $\hat{\theta}_{B L}$ denote the Bayesian estimates of $\theta$ under SEL and LINEX loss, respectively. 
Table 2. Estimates, MSE, and $95 \%$ CIs and CRIs of MLEs, Bayesian, and E-Bayesian methods for $R(t=1.3)$ under LINEX and SEL functions when $\theta=1.22, a=0.8, b=0.7, h=1.5, c=1$.

\begin{tabular}{|c|c|c|c|c|c|c|c|c|c|c|c|}
\hline \multirow[t]{2}{*}{$(n, r)$} & \multirow[t]{2}{*}{$\left(T_{1}, T_{2}\right)$} & \multirow[t]{2}{*}{ Criteria } & \multirow[t]{2}{*}{$\hat{\mathbf{R}}_{M L E}$} & \multicolumn{4}{|c|}{ SEL Function } & \multicolumn{4}{|c|}{ LINEX Loss } \\
\hline & & & & $\hat{R}_{B S}$ & $\hat{R}_{E B S 1}$ & $\hat{R}_{E B S 2}$ & $\hat{R}_{E B S 3}$ & $\hat{R}_{B L}$ & $\hat{R}_{E B L 1}$ & $\hat{R}_{E B L 2}$ & $\hat{R}_{E B L 3}$ \\
\hline \multirow[t]{10}{*}{$(25,15)$} & $(0.7,1.5)$ & Mean & 0.307776 & 0.30712 & 0.27518 & 0.24078 & 0.25282 & 0.30532 & 0.27373 & 0.23967 & 0.2516 \\
\hline & & MSE & 0.011812 & 0.00825 & 0.00359 & 0.00086 & 0.00154 & 0.00793 & 0.00342 & 0.00081 & 0.00146 \\
\hline & & Lower & 0.174502 & 0.2112 & 0.2094 & 0.1607 & 0.0792 & 0.1096 & 0.1577 & 0.0764 & 0.1066 \\
\hline & & Upper & 0.44105 & 0.4030 & 0.4013 & 0.3897 & 0.4023 & 0.3961 & 0.3898 & 0.4030 & 0.3966 \\
\hline & & Length & 0.266548 & 0.1917 & 0.1919 & 0.2290 & 0.3231 & 0.2865 & 0.2321 & 0.3266 & 0.2901 \\
\hline & $(1.5,2)$ & Mean & 0.318297 & 0.29404 & 0.26346 & 0.23052 & 0.24205 & 0.29241 & 0.26215 & 0.22952 & 0.24095 \\
\hline & & MSE & 0.010628 & 0.00623 & 0.0025 & 0.00059 & 0.00101 & 0.00598 & 0.00238 & 0.00056 & 0.00095 \\
\hline & & Lower & 0.252122 & 0.2031 & 0.2015 & 0.1546 & 0.0764 & 0.1055 & 0.1518 & 0.0738 & 0.1028 \\
\hline & & Upper & 0.384472 & 0.3849 & 0.3834 & 0.3724 & 0.3847 & 0.3786 & 0.3725 & 0.3853 & 0.3791 \\
\hline & & Length & 0.132349 & 0.1818 & 0.1819 & 0.2178 & 0.3083 & 0.2731 & 0.2206 & 0.3115 & 0.2764 \\
\hline \multirow[t]{10}{*}{$(45,30)$} & $(0.7,1.5)$ & Mean & 0.302633 & 0.30199 & 0.27058 & 0.23676 & 0.2486 & 0.30094 & 0.26974 & 0.23612 & 0.24789 \\
\hline & & MSE & 0.009276 & 0.0071 & 0.00287 & 0.00053 & 0.00109 & 0.00692 & 0.00278 & 0.00051 & 0.00105 \\
\hline & & Lower & 0.193104 & 0.2288 & 0.2278 & 0.1750 & 0.0895 & 0.1209 & 0.1731 & 0.0877 & 0.1191 \\
\hline & & Upper & 0.412162 & 0.3751 & 0.3741 & 0.3662 & 0.3841 & 0.3763 & 0.3664 & 0.3845 & 0.3767 \\
\hline & & Length & 0.219058 & 0.1463 & 0.1463 & 0.1912 & 0.2946 & 0.2554 & 0.1933 & 0.2968 & 0.2576 \\
\hline & $(1.5,2)$ & Mean & 0.300882 & 0.28955 & 0.25944 & 0.22701 & 0.23836 & 0.28859 & 0.25867 & 0.22642 & 0.23771 \\
\hline & & MSE & 0.008729 & 0.00514 & 0.0018 & 0.00025 & 0.00056 & 0.0050 & 0.00174 & 0.00024 & 0.23771 \\
\hline & & Lower & 0.203186 & 0.2194 & 0.2184 & 0.1678 & 0.0858 & 0.1159 & 0.1660 & 0.0842 & 0.1142 \\
\hline & & Upper & 0.398578 & 0.3597 & 0.3587 & 0.3511 & 0.3682 & 0.3608 & 0.3513 & 0.3687 & 0.3612 \\
\hline & & Length & 0.195393 & 0.1402 & 0.1403 & 0.1833 & 0.2825 & 0.2448 & 0.1853 & 0.2845 & 0.2469 \\
\hline \multirow[t]{10}{*}{$(60,45)$} & $(0.7,1.5)$ & & 0.298925 & 0.28809 & 0.25813 & 0.22586 & 0.23715 & 0.28737 & 0.25755 & 0.22542 & 0.23667 \\
\hline & & MSE & 0.008271 & 0.00491 & 0.00168 & 0.00022 & 0.0005 & 0.00481 & 0.00164 & 0.00022 & 0.000479 \\
\hline & & Lower & 0.204471 & 0.2275 & 0.2268 & 0.1738 & 0.0897 & 0.1204 & 0.1724 & 0.0885 & 0.1191 \\
\hline & & Upper & 0.393379 & 0.3486 & 0.3479 & 0.3425 & 0.3620 & 0.3539 & 0.3427 & 0.3624 & 0.3543 \\
\hline & & Length & 0.188907 & 0.1211 & 0.1212 & 0.1687 & 0.2724 & 0.2335 & 0.1703 & 0.2739 & 0.2352 \\
\hline & $(1.5,2)$ & Mean & 0.29833 & 0.28406 & 0.25451 & 0.2227 & 0.23383 & 0.28336 & 0.25396 & 0.22227 & 0.23337 \\
\hline & & MSE & 0.007858 & 0.00436 & 0.00141 & 0.00019 & 0.00038 & 0.00427 & 0.00137 & 0.00019 & 0.00037 \\
\hline & & Lower & 0.211413 & 0.2245 & 0.2238 & 0.1715 & 0.0885 & 0.1188 & 0.1702 & 0.0873 & 0.1175 \\
\hline & & Upper & 0.385247 & 0.3436 & 0.3429 & 0.3376 & 0.3569 & 0.3489 & 0.3378 & 0.3572 & 0.3492 \\
\hline & & Length & 0.173834 & 0.1190 & 0.1191 & 0.1661 & 0.2684 & 0.2301 & 0.1676 & 0.2699 & 0.2316 \\
\hline \multirow[t]{10}{*}{$(80,65)$} & $(0.7,1.5)$ & Mean & 0.298976 & 0.28424 & 0.25468 & 0.22285 & 0.23399 & 0.28371 & 0.25425 & 0.22252 & 0.23363 \\
\hline & & MSE & 0.007187 & 0.00424 & 0.00131 & 0.0001 & 0.00029 & 0.00417 & 0.00128 & 0.0001 & 0.00028 \\
\hline & & Lower & 0.233718 & 0.2318 & 0.2313 & 0.1765 & 0.0916 & 0.1224 & 0.1755 & 0.0907 & 0.1214 \\
\hline & & Upper & 0.364234 & 0.3367 & 0.3362 & 0.3328 & 0.3541 & 0.3456 & 0.333 & 0.3544 & 0.3458 \\
\hline & & Length & 0.130516 & 0.1049 & 0.1049 & 0.1563 & 0.2625 & 0.2232 & 0.1576 & 0.2637 & 0.2244 \\
\hline & $(1.5,2)$ & Mean & 0.300122 & 0.2825 & 0.25312 & 0.22148 & 0.23256 & 0.28198 & 0.25271 & 0.22116 & 0.23221 \\
\hline & & MSE & 0.006783 & 0.00391 & 0.00111 & 0.00003 & 0.00018 & 0.00384 & 0.00108 & 0.00003 & 0.00017 \\
\hline & & Lower & 0.25815 & 0.2310 & 0.2305 & 0.1759 & 0.0913 & 0.1219 & 0.1748 & 0.0904 & 0.1210 \\
\hline & & Upper & 0.342094 & 0.3340 & 0.3335 & 0.3304 & 0.3517 & 0.3432 & 0.3306 & 0.3520 & 0.3434 \\
\hline & & Length & 0.0839436 & 0.1030 & 0.1030 & 0.1545 & 0.2604 & 0.2212 & 0.1557 & 0.2616 & 0.2225 \\
\hline
\end{tabular}

Where $\hat{R}_{E B S 1}, \hat{R}_{E B S 3}$ and $\hat{R}_{E B S 2}$ are the E-Bayesian estimates of $R(t)$ relative to SEL based on $p_{1}(a, b), p_{2}(a, b)$ and $p_{3}(a, b)$, respectively, $\hat{R}_{E B L 1}, \hat{R}_{E B L 2}$ and $\hat{R}_{E B L 3}$ are the E-Bayesian estimates of $R(t)$ relative to LINEX based on $p_{1}(a, b), p_{2}(a, b)$ and $p_{3}(a, b)$, respectively. In addition, $\hat{R}_{B S}$ and $\hat{R}_{B L}$ denote the Bayesian estimates of $R(t)$ under SEL and LINEX loss, respectively.

\section{Illustrative Example (Real Data Set)}

An illustrative example is provided to investigate the performance of proposed methods and clarify how they behave in practice. These data were reported by [21], representing minority electron mobility for $G a_{1-x} A l_{x} A s$. Two data sets at the mole fractions of 0.25 and 0.30 were used by [22], 
who stated that Burr-X distribution presents a good fit for Sets 1 and 2 of data. Each set contains 21 observations, as given below:

Data Set 1 ( mole fraction 0.25): 0.7948, 0.7007, 0.6292, 3.051, 2.779, 2.604, 2.371, 1.525, 1.296, 1.154, $2.214,2.045,1.715,1.016,0.6175,0.6449,0.8881,1.115,1.397,1.506$ and 1.528 .

Data Set 2 ( mole fraction 0.30): 2.092, 1.959, 1.814, 2.658, 2.434, 2.288, 1.530, 1.366, 1.165, 1.041, $1.002,1.250,1.347,0.9198,0.7241,0.6403,0.576,0.5647,0.5873,0.8013$, and 1.368 .

By using generalized Type-II HCS, taking $n=21, r=15, \theta=2$ and $R(t=1.2)$, we observe the following cases, for data Set 1 :

- When $T_{1}=0.7$ and $T_{2}=1.5$, we observe that $Y_{r: n}=1.715>T_{2}$, hence the experiment is terminated at random time $T_{2}=1.5$, i.e., only 11 items fail at a random time $T_{2}=1.5$.

- When $T_{1}=0.9$ and $T_{2}=2$, we observe that $T_{1}<Y_{r: n}=1.715<T_{2}$, hence the experiment is finished at random time $Y_{r: n}=1.715$, i.e., 15 items fail at random time $Y_{r: n}=1.715$.

For data Set 2:

- When $T_{1}=0.7$ and $T_{2}=1.5$, we observe that $Y_{r: n}=1.53>T_{2}$, hence the experiment is terminated at random time $T_{2}=1.5$. Therefore, only 14 items fail out of 21 items at random time $T_{2}=1.5$.

- When $T_{1}=0.9$ and $T_{2}=1.7$, we observe that $T_{1}<Y_{r: n}=1.153<T_{2}$, hence the experiment is terminated at random time $Y_{r: n}=1.53$, i.e., 15 items are obtained out of 21 items at random time $Y_{r: n}=1.53$.

With respect to real data Sets 1 and 2, all estimates are obtained according to the same procedure as before and results are displayed in Tables 3-6. Tables 3 and 5, give average estimates, MSE, and 95\% CIs and CRIs of MLEs, Bayesian, and E-Bayesian estimates for $\theta$ under LINEX and SEL functions belong to real data Sets 1 and 2, respectively. Tables 4 and 6, display average estimates, MSE and 95\% CIs and CRIs of MLEs, Bayesian, and E-Bayesian estimates of $R(t=1.2)$ based on LINEX and SEL functions belong to real data Sets 1 and 2, respectively.

Table 3. For real data Set 1: Estimates, MSE and 95\%CIs and CRIs of MLEs, Bayesian and E-Bayesian methods for $\theta$ under LINEX and SEL functions when $\theta=2, a=0.8, b=0.7, h=1.5, c=1$.

\begin{tabular}{|c|c|c|c|c|c|c|c|c|c|c|c|}
\hline \multirow[t]{2}{*}{$(n, r)$} & \multirow[t]{2}{*}{$\left(T_{1}, T_{2}\right)$} & \multirow[t]{2}{*}{ Criteria } & \multirow[t]{2}{*}{$\hat{\theta}_{M L E}$} & \multicolumn{4}{|c|}{ SEL Function } & \multicolumn{4}{|c|}{ LINEX Loss } \\
\hline & & & & $\hat{\theta}_{B S}$ & $\hat{\theta}_{E B S 1}$ & $\hat{\theta}_{E B S 2}$ & $\hat{\theta}_{E B S 3}$ & $\hat{\theta}_{B L}$ & $\hat{\theta}_{E B L 1}$ & $\hat{\theta}_{E B L 2}$ & $\hat{\theta}_{E B L 3}$ \\
\hline \multirow[t]{10}{*}{$(21,15)$} & $(0.7,1.5)$ & Mean & 2.71786 & 2.81461 & 2.52189 & 2.20665 & 2.31698 & 2.31698 & 2.32603 & 2.0548 & 2.1503 \\
\hline & & MSE & 0.7076 & 0.6636 & 0.27238 & 0.04271 & 0.10049 & 0.32886 & 0.10631 & 0.00301 & 0.0226 \\
\hline & & Lower & 1.66523 & 1.6376 & 1.3051 & 1.2125 & 0.5317 & 0.7883 & 0.8086 & 0.1566 & 0.3951 \\
\hline & & Upper & 3.77049 & 3.9916 & 3.8418 & 3.8313 & 3.8816 & 3.8456 & 3.8434 & 3.953 & 3.9055 \\
\hline & & Length & 2.10526 & 2.3541 & 2.5368 & 2.6188 & 3.3498 & 3.0573 & 3.0348 & 3.7964 & 3.5104 \\
\hline & $(0.9,1.7)$ & Mean & 2.83501 & 2.7219 & 2.43882 & 2.13397 & 2.24067 & 2.49547 & 2.25497 & 1.99146 & 2.08423 \\
\hline & & MSE & 0.70233 & 0.52121 & 0.19262 & 0.01799 & 0.05797 & 0.24558 & 0.06508 & 0.00013 & 0.00715 \\
\hline & & Lower & 2.68764 & 1.5827 & 1.2729 & 1.1717 & 0.5135 & 0.7616 & 0.7937 & 0.1618 & 0.3931 \\
\hline & & Upper & 2.98238 & 3.8611 & 3.7181 & 3.706 & 3.7544 & 3.7197 & 3.7162 & 3.8211 & 3.7753 \\
\hline & & Length & 0.294742 & 2.2784 & 2.4452 & 2.5343 & 3.2408 & 2.958 & 2.9226 & 3.6592 & 3.3822 \\
\hline
\end{tabular}


Table 4. For real data Set 1: Estimates, MSE and 95\%CIs and CRIs of MLEs, Bayesian and E-Bayesian methods for $R(t=1.2)$ under LINEX and SEL functions, $\theta=2, a=0.8, b=0.7, h=1.5, c=1$.

\begin{tabular}{|c|c|c|c|c|c|c|c|c|c|c|c|}
\hline \multirow[t]{2}{*}{$(n, r)$} & \multirow[t]{2}{*}{$\left(T_{1}, T_{2}\right)$} & \multirow[t]{2}{*}{ Criteria } & \multirow[t]{2}{*}{$\hat{R}_{M L E}$} & \multicolumn{4}{|c|}{ SEL Function } & \multicolumn{4}{|c|}{ LINEX Loss } \\
\hline & & & & $\hat{R}_{B S}$ & $\hat{R}_{E B S 1}$ & $\hat{R}_{E B S 2}$ & $\hat{R}_{E B S 3}$ & $\hat{R}_{B L}$ & $\hat{R}_{E B L 1}$ & $\hat{R}_{E B L 2}$ & $\hat{R}_{E B L 3}$ \\
\hline \multirow[t]{10}{*}{$(21,15)$} & $(0.7,1.5)$ & Mean & 0.517062 & 0.52677 & 0.47198 & 0.41299 & 0.43363 & 0.52255 & 0.4686 & 0.41039 & 0.43078 \\
\hline & & MSE & 0.013175 & 0.01189 & 0.00294 & 0.00002 & 0.00025 & 0.01099 & 0.00259 & 0.00005 & 0.00017 \\
\hline & & Lower & 0.379033 & 0.3799 & 0.3755 & 0.2901 & 0.1460 & 0.1994 & 0.2827 & 0.1391 & 0.1921 \\
\hline & & Upper & 0.655091 & 0.6736 & 0.6696 & 0.6539 & 0.6800 & 0.6679 & 0.6545 & 0.6817 & 0.6694 \\
\hline & & Length & 0.276057 & 0.2936 & 0.2941 & 0.3638 & 0.5340 & 0.4685 & 0.3718 & 0.5425 & 0.4773 \\
\hline & $(0.9,1.7)$ & Mean & 0.535322 & 0.51515 & 0.46157 & 0.40387 & 0.42407 & 0.5110 & 0.45824 & 0.40133 & 0.42126 \\
\hline & & MSE & 0.013907 & 0.00949 & 0.00192 & 0.00019 & 0.00004 & 0.0087 & 0.00164 & 0.00027 & 0.000013 \\
\hline & & Lower & 0.517135 & 0.3695 & 0.3652 & 0.282 & 0.1416 & 0.1937 & 0.2748 & 0.1349 & 0.1866 \\
\hline & & Upper & 0.55350 & 0.6608 & 0.6568 & 0.6411 & 0.6661 & 0.6544 & 0.6417 & 0.6677 & 0.6559 \\
\hline & & Length & 0.0363737 & 0.2912 & 0.2917 & 0.3591 & 0.5245 & 0.4608 & 0.3668 & 0.5328 & 0.4693 \\
\hline
\end{tabular}

Table 5. For real data Set 2: Estimates, MSE and 95\%CIs and CRIs of MLEs, Bayesian, and E-Bayesian methods for $\theta$ under LINEX and SEL functions when $\theta=2, a=0.8, b=0.7, h=1.5, c=1$.

\begin{tabular}{|c|c|c|c|c|c|c|c|c|c|c|c|}
\hline \multirow[t]{2}{*}{$(n, r)$} & \multirow[t]{2}{*}{$\left(T_{1}, T_{2}\right)$} & \multirow[t]{2}{*}{ Criteria } & \multirow[t]{2}{*}{$\hat{\theta}_{M L E}$} & \multicolumn{4}{|c|}{ SEL Function } & \multicolumn{4}{|c|}{ LINEX Loss } \\
\hline & & & & $\hat{\theta}_{B S}$ & $\hat{\theta}_{E B S 1}$ & $\hat{\theta}_{E B S 2}$ & $\hat{\theta}_{E B S 3}$ & $\hat{\theta}_{B L}$ & $\hat{\theta}_{E B L 1}$ & $\hat{\theta}_{E B L 2}$ & $\hat{\theta}_{E B L 3}$ \\
\hline \multirow[t]{10}{*}{$(21,15)$} & $(0.7,1.5)$ & Mean & 2.30514 & 2.43127 & 2.17842 & 1.90612 & 2.00142 & 2.24881 & 2.03042 & 1.79153 & 1.87559 \\
\hline & & MSE & 0.31672 & 0.18603 & 0.03186 & 0.00884 & 0.00003 & 0.06194 & 0.00095 & 0.04348 & 0.0155 \\
\hline & & Lower & 1.32817 & 1.4141 & 1.1706 & 1.0469 & 0.4589 & 0.6806 & 0.7451 & 0.1769 & 0.3853 \\
\hline & & Upper & 3.28211 & 3.4485 & 3.3271 & 3.310 & 3.3533 & 3.3223 & 3.3157 & 3.4062 & 3.3659 \\
\hline & & Length & 1.95393 & 2.0344 & 2.1565 & 2.2631 & 2.8943 & 2.6417 & 2.5706 & 3.2292 & 2.9806 \\
\hline & $(0.9,1.7)$ & Mean & 2.25925 & 2.39822 & 2.1488 & 1.8802 & 1.97421 & 2.22801 & 2.00479 & 1.77471 & 1.85176 \\
\hline & & MSE & 0.18272 & 0.15868 & 0.02223 & 0.01442 & 0.00074 & 0.05203 & 0.0001 & 0.05078 & 0.02204 \\
\hline & & Lower & 1.55708 & 1.3943 & 1.1582 & 1.0322 & 0.4524 & 0.6714 & 0.7389 & 0.1748 & 0.3838 \\
\hline & & Upper & 2.96142 & 3.4021 & 3.2832 & 3.2654 & 3.308 & 3.2923 & 3.2707 & 3.3746 & 3.3197 \\
\hline & & Length & 1.40434 & 2.0078 & 2.1250 & 2.2332 & 2.8557 & 2.6209 & 2.5318 & 3.1998 & 2.936 \\
\hline
\end{tabular}

Table 6. For real data Set 2: Estimates, MSE, and 95\%CIs and CRIs of MLEs, Bayesian, and E-Bayesian methods for $R(t=1.2)$ under LINEX and SEL functions, $\theta=1.22, a=0.8, b=0.7, h=1.5, c=1$.

\begin{tabular}{|c|c|c|c|c|c|c|c|c|c|c|c|}
\hline \multirow[t]{2}{*}{$(n, r)$} & \multirow[t]{2}{*}{$\left(T_{1}, T_{2}\right)$} & \multirow[t]{2}{*}{ Criteria } & \multirow[t]{2}{*}{$\hat{R}_{M L E}$} & \multicolumn{4}{|c|}{ SEL Function } & \multicolumn{4}{|c|}{ LINEX Loss } \\
\hline & & & & $\hat{R}_{B S}$ & $\hat{R}_{E B S 1}$ & $\hat{R}_{E B S 2}$ & $\hat{R}_{E B S 3}$ & $\hat{R}_{B L}$ & $\hat{R}_{E B L 1}$ & $\hat{R}_{E B L 2}$ & $\hat{R}_{E B L 3}$ \\
\hline \multirow[t]{10}{*}{$(21,15)$} & $(0.7,1.5)$ & Mean & 0.50136 & 0.47678 & 0.4272 & 0.3738 & 0.39249 & 0.47292 & 0.4241 & 0.37143 & 0.38987 \\
\hline & & MSE & 0.012054 & 0.00349 & 0.00009 & 0.00193 & 0.00064 & 0.00305 & 0.00004 & 0.00214 & 0.000776 \\
\hline & & Lower & 0.354413 & 0.3362 & 0.3322 & 0.2563 & 0.1278 & 0.1756 & 0.2497 & 0.1216 & 0.1690 \\
\hline & & Upper & 0.648307 & 0.6173 & 0.6137 & 0.5981 & 0.6198 & 0.6094 & 0.5985 & 0.6212 & 0.6107 \\
\hline & & Length & 0.293894 & 0.2811 & 0.2815 & 0.3418 & 0.4919 & 0.4338 & 0.3488 & 0.4996 & 0.4417 \\
\hline & $(0.9,1.7)$ & Mean & 0.455001 & 0.47222 & 0.42311 & 0.37022 & 0.38873 & 0.4684 & 0.42004 & 0.36787 & 0.38614 \\
\hline & & MSE & 0.003422 & 0.00297 & 0.00003 & 0.00226 & 0.00084 & 0.00257 & 0.00001 & 0.00249 & 0.00099 \\
\hline & & Lower & 0.36187 & 0.3324 & 0.3284 & 0.2533 & 0.1262 & 0.1735 & 0.2468 & 0.1201 & 0.1670 \\
\hline & & Upper & 0.548132 & 0.6121 & 0.6084 & 0.5929 & 0.6142 & 0.6040 & 0.5933 & 0.6156 & 0.6053 \\
\hline & & Length & 0.18626 & 0.2797 & 0.2801 & 0.3395 & 0.4880 & 0.4305 & 0.3465 & 0.4955 & 0.4383 \\
\hline
\end{tabular}

\section{Concluding Remarks}

In this article, we used the Bayesian and E-Bayesian approaches and the ML method for estimating the parameter $\theta$ and the reliability function $R(t)$ of one-parameter Burr-X distribution under generalized Type-II HCS. It is noted that Bayesian estimators cannot be derived analytically, so we applied the MCMC method to derive estimates of the parameter $\theta$ and the reliability function $R(t)$. Based on SEL and LINEX loss functions, Bayesian and E-Bayesian estimates are derived. Also, 
CIs of MLEs and CRIs of Bayesian and E-Bayesian estimates are constructed. Furthermore, an example of real data testing is provided for illustration.

From Tables 1 and 2, one can observe that the MSEs decrease when the sample sizes $n, T_{1}$, and $T_{2}$ increase. Also, the length of CIs and CRIs decreases when time points $T_{1}$ and $T_{2}$ increase for constant sample size $n$. The MSE of E-Bayesian estimator for the parameter $\theta$ and the reliability function $R(t)$ is the smallest compared to the MSE of MLEs and Bayesian estimates. Furthermore, the estimator by using LINEX loss function is more efficient than the estimator based on SEL function in terms of MSE. Generally, The LINEX loss function is better than SEL function in most cases. Ref. [23] compared the difference between the LINEX and SEL functions and stated that the LINEX loss is more appropriate than the SEL function. For the shape parameter $h$ of LINEX close to zero, it is approximately SEL and, therefore, almost symmetric. Based on the above results, we can state that a large sample size $n$ gives a better estimate with a smaller MSE. Finally, we can conclude that the E-Bayesian method is more convenient and efficient when compared with ML and Bayesian methods. The prior PDFs affect the E-Bayesian estimation because of selecting the restriction of $0<a<1$ and $0<b<c$.

From Tables 3-6, we observe that the E-Bayesian method is more efficient than ML, and Bayesian methods in the sense of having smaller MSE. On the other hand, MSEs of all estimators and the length of CIs and CRIs decrease when time points $T_{1}$ and $T_{2}$ increase, i.e., the proposed methods perform efficiently in the application as well.

Author Contributions: This work is written and prepared by A. Rabie under the supervision and review of J. Li.

Funding: This work was substantially supported by the National Natural Sciences Foundations of China (No. 11771452, No. 11571372) and Natural Sciences Foundations of Hunan (No. 2017JJ2328).

Acknowledgments: The authors would like to thank the associate editor, the editor and the referees for their constructive comments and suggestions that improved the content and the style of this article.

Conflicts of Interest: The authors declare no conflict of interest.

\section{Appendix A}

$$
\frac{\partial \ell}{\partial \theta}= \begin{cases}\frac{D_{1}}{\theta}-\frac{n-D_{1}}{1-W_{T_{1}}^{\theta}} W_{T_{1}}^{\theta} \ln W_{T_{1}}-\sum_{i=1}^{D_{1}} \ln W_{y_{i: n}}, & D_{1}=r, r+1, \cdots, n, \\ \frac{r}{\theta}-\frac{n-r}{1-W_{y_{r: n}}^{\theta}} W_{y_{r: n}}^{\theta} \ln W_{y_{r: n}}-\sum_{i=1}^{r} \ln W_{y_{i: n} n^{\prime}}, & D_{1}=0,1, \cdots, r-1, D_{2}=r, \\ \frac{D_{2}}{\theta}-\frac{n-D_{2}}{1-W_{T_{2}}^{\theta}} W_{T_{2}}^{\theta} \ln W_{T_{2}}-\sum_{i=1}^{D_{2}} \ln W_{y_{i: n} n^{\prime}}, & D_{2}=0,1, \cdots, r-1,\end{cases}
$$

where $W_{y_{i: n}}=1-e^{-y_{i: n}^{2}}, W_{T_{1}}=1-e^{-T_{1}^{2}}$ and $W_{T_{2}}=1-e^{-T_{2}^{2}}$.

\section{References}

1. Epstein, B. Truncated life tests in exponential case. Ann. Math. Stat. 1954, 25, 555-564. [CrossRef]

2. Chandrasekar, B.; Childs, A.; Balakrishnan, N. Exact likelihood inference for the exponential distribution under generalized Type-I and Type-II hybrid censoring. Naval Res. Logist. 2004, 51, 994-1004. [CrossRef]

3. Balakrishnan, N.; Kundu, D. Hybrid censoring models, inferential results and applications. Comput. Stat. Data Anal. 2013, 57, 166-209. [CrossRef]

4. Shafay, A.R. Bayesian estimation and prediction based on generalized Type-II hybrid censored sample. J. Stat. Comput. Simul. 2015, 32, 641-661. [CrossRef]

5. Mahmoud, M.A.W.; Ghazal, M.G.M. Estimations from the exponentiated rayleigh distribution based on generalized Type-II hybrid censored data. J. Egypt. Math. Soc. 2017, 25, 71-78. [CrossRef]

6. Burr, W.I. Cumulative frequency distribution. Ann. Math. Stat. 1942, 13, 215-232. [CrossRef]

7. Pandiyan; Chandrabose, S.; Pukazhenthi; Subramanian. A stochastic model for aids patients using Burr Type X distribution. Int. J. Adv. Innov. Res. 2015, 4, 148-150.

8. Ahmad, A.A.; El-Adll, M.E.; AlOafi, T.W. Estimation under Burr type $X$ distribution based on doubly type II censored sample of dual generalized order statistics. J. Egypt. Math. Soc. 2015, 23, 391-396. [CrossRef] 
9. Aludaat, K.M.; Alodat, M.T.; Alodat, T.T. Parameter estimation of Burr type X distribution for grouped data. Appl. Math. Sci. 2008, 2, 415-423.

10. Mousa, M.A. Inferences and prediction for the Burr type X model based on records. J. Theor. Appl. Stat. 2007, $41,415-425$.

11. Sindhu, T.N.; Aslam, M. On the parameter of Burr Type X under Bayesian principles. Int. J. Math. Comput. Sci. 2014, 8, 185-192.

12. Rabie, A.; Li, J. E-Bayesian estimation for Burr-X distribution based on Type-I hybrid censoring scheme. IAENG Int. J. Appl. Math. 2018, 48, 244-250. [CrossRef]

13. Zellner, A. Bayesian estimation and prediction using asymmetric loss functions. J. Am. Stat. Assoc. 1986, 18, 446-451. [CrossRef]

14. Han, M. The structure of hierarchical prior distribution and its applications. Chin. Oper. Res. Manag. Sci. 1997, 6, 31-40.

15. Han, M. E-Bayesian estimation and hierarchical Bayesian estimation of failure rate. Appl. Math. Model. 2009, 33, 1915-1922. [CrossRef]

16. Jaheen, Z.F.; Okasha, H.M. E-Bayesian estimation for the Burr Type XII model based on Type II censoring. Appl. Math. Model. 2011, 35, 4730-4437. [CrossRef]

17. Okasha, H.M. E-Bayesian estimation for the Lomax distribution on Type-II censored data. J. Egypt. Math. Soc. 2014, 22, 489-495. [CrossRef]

18. Rabie, A.; Li, J. E-Bayesian estimation for the Burr-X distribution based on generalized Type I hybrid censoring scheme. Am. J. Math. Manag. Sci. 2019. [CrossRef]

19. Hastings, W.K. Monte Carlo sampling methods using Markov chains and their applications. Biometrika 1970, 57, 97-109. [CrossRef]

20. Cowles, M.K.; Carlin, B.P. Markov chain Monte Carlo diagnostics: A comparative review. J. Am. Stat. Assoc. 1995, 91, 883-904. [CrossRef]

21. Bennett, H.S.; Filliben, J.J. A Systematic approach for multidimensional, closed-form analytic modeling: Minority electron mobilities in $G a_{1-x} A l_{x}$ As hetero structures. J. Res. Natl. Inst. Stand. Technol. 2000, 105, 441-452. [CrossRef] [PubMed]

22. Tarvirdizade, B.; Gharehchobogh, H.K. Inference on $\operatorname{Pr}(X>Y)$ Based on Record Values from the Burr Type $X$ Distribution. Hacet. J. Math. Stat. 2016, 45, 267-278. [CrossRef]

23. Soliman, A.A. Comparison of linex and quadratic bayes estimators for the rayleigh distribution. Commun. Stat. Theory Methods 2000, 29, 95-107. [CrossRef] 